\title{
Impacto de la implementación de Código Infarto en pacientes con infarto agudo de miocardio con elevación del segmento ST en el Hospital de Cardiología del Centro Médico Nacional Siglo XXI
}

\author{
Gabriela Borrayo Sánchez, Héctor Álvarez Covarrubias², Gilberto Pérez Rodríguez', \\ Efraín Arizmendi Uribe ${ }^{2}$, Erick Ramírez-Arias², Martín Rosas Peralta ${ }^{1}$ y Jesús Arriaga Dávila ${ }^{3}$ \\ ${ }^{1}$ Coordinación de Unidades Médicas de Alta Especialidad; ${ }^{2}$ Hospital de Cardiología, Centro Médico Nacional Siglo XXI, Instituto Mexicano del \\ Seguro Social; ${ }^{3}$ Dirección de Prestaciones Médicas, Ciudad de México, México
}

\begin{abstract}
Resumen
Objetivo: Evaluar el impacto de la implementación de la estrategia Código Infarto en pacientes con infarto agudo de miocardio (IAM) durante la hospitalización. Métodos: Incluimos pacientes consecutivos con IAM con elevación del segmento ST de $\leq 12$ horas de evolución, antes (grupo I) y después (grupo II) del Código Infarto. Se analizaron los tiempos de atención y los eventos cardiovasculares mayores durante la hospitalización. Resultados: Incluimos 1,227 pacientes, 919 hombres (75\%) y 308 mujeres (25\%), con una edad promedio de $64 \pm 11$ años. Entre los grupos I y II cambió la reperfusión con intervención coronaria percutánea (16.6 a 42.6\%), terapia fibrinolítica (39.3 a 25\%) y no reperfusión (44 a 32.6\%; $p<0.0001$ ). Los tiempos de atención disminuyeron significativamente (puerta-aguja 92 a 72 minutos, $p=0.004$; puerta-balón 140 a 92 minutos, $p<0.0001$ ). Disminuyeron la falla renal (24.6 contra $17.9 \%, p=0.006)$, las complicaciones mayores (35.3 a $29.3 \%)$ y las muertes (21 contra 12\%; odds ratio: 0.52; intervalo de confianza del 95\%: 0.38-0.71; $p=0.004$ ). Conclusión: El Código Infarto mejoró el tratamiento y los tiempos de atención, y disminuyó las complicaciones y las muertes en estos pacientes.
\end{abstract}

PALABRAS CLAVE: Infarto agudo de miocardio. Código Infarto.

\begin{abstract}
Objective: To evaluate the impact of the implementation of the Infarction Code strategy in patients with acute myocardial infarction diagnosis. Methods: Consecutive patients with ST-elevation acute myocardial infarction $\leq 12$ hours of evolution, were included in the infarction code strategy, before (Group I) and after (Group II). Times of medical attention and major cardiovascular events during hospitalization were analyzed. Results: 1227 patients were included, 919 men (75\%) and 308 women (25\%) with an average age of $62 \pm 11$ years. Among Group I and Group II, percutaneous coronary intervention reperfusion therapy changed (16.6\% to $42.6 \%)$, fibrinolytic therapy (39.3\% to $25 \%)$, and patients who did not receive any form of reperfusion therapy (44\% to 32.6\%; $p<0.0001)$. Times of medical attention decreased significantly (door-to-needle time decreased from 92 to 72 minutes, $p=0.004$; door-to-balloon time decreased from 140 to 92 minutes, $p<0.0001$ ). Kidney failure $(24.6 \%$ vs. $17.9 \%$; $p=0.006)$, major complications (35.3\% to $29.3 \%)$, and death $(21 \%$ vs. $12 \%$; odds ratio: 0.52; 95\% confidence interval: 0.38-0.71; $p=0.004)$. also decreased. Conclusion: The Infarction Code strategy improved treatment, times of medical attention and decreased complications and death in these patients.
\end{abstract}

KEY WORDS: Acute myocardial infarction. Infarction code.

Correspondencia:

Gabriela Borrayo Sánchez

Durango 289, tercer piso

Col. Roma, Del. Cuauhtémoc

Fecha de recepción: 07-08-2017

C.P. 06700, Ciudad de México, México

Fecha de aceptación: 07-09-2017

E-mail: gabriela.borrayo@imss.gob.mx.

DOI://dx.doi.org/10.24875/GMM.M17000002

Gac Med Mex. 2017;153;Sup 2:S13-S17 Contents available at PubMed www.anmm.org.mx 


\section{Introducción}

La Organización para la Cooperación y el Desarrollo Económicos ${ }^{1}$ colocó en 2015 a México en el primer lugar en mortalidad por infarto agudo de miocardio (IAM) en mayores de 45 años en los primeros 30 días; el Instituto Nacional de Estadística y Geografía reportó más de 121 muertes por esta causa, el 70\% por cardiopatía isquémica, principalmente IAM ${ }^{2}$. La reperfusión de la arteria causante es el objetivo principal.

Existe evidencia a favor de la intervención coronaria percutánea (ICP) comparada con la terapia fibrinolítica (TF); como se demostró en un metaanálisis, menor mortalidad, reinfarto e isquemia recurrente ${ }^{3}$. Sin embargo, en las primeras 2 horas se pueden considerar equivalentes ${ }^{4,5}$, por lo que la estrategia implementada en el tiempo más cercano al inicio de los síntomas puede ser benéfica. Las guías de práctica clínica actuales sugieren que todas las comunidades deben crear o mantener un sistema regionalizado para el tratamiento del IAM con elevación el segmento ST (IAM CEST), con una mejora en la calidad de atención en los servicios de urgencias ${ }^{6,7}$.

Los estudios de vida real indican que este proceso requiere tiempo; por mencionar algunos, en el estudio RENASCA IMSS ${ }^{8}$, con 2389 pacientes, el 65\% eran de alto riesgo, pero el 50\% no recibían ninguna estrategia de reperfusión, el $42 \%$ TF y solo el $8 \%$ ICP. Algo similar mostró el Registro CREATE ${ }^{9}$ de la India, con 20,468 pacientes, de los que el $8 \%$ recibió ICP y el $58.5 \%$ recibió TF (96.3\% con estreptocinasa); el $60 \%$ con IAM CEST mostró retraso en el tiempo para recibir atención (mediana 360 minutos).

Ante esta problemática, el Instituto Mexicano del Seguro Social (IMSS) crea el primer protocolo de atención para los servicios de urgencias, llamado Código Infarto ${ }^{10}$, con el objetivo de garantizar el diagnóstico y el tratamiento con ICP en menos de 90 minutos o con TF en menos de 30 minutos en pacientes que demandan atención de urgencias por IAM CEST.

\section{Métodos}

Se incluyeron pacientes consecutivos que ingresaran a los servicios de urgencias, de ambos sexos, entre 18 y 90 años de edad, con diagnóstico de IAM CEST, de acuerdo con los criterios diagnósticos internacionales ACC/AHA/ESC (American College of Cardiology/American Heart Association/European Society of Cardiology), atendidos en los servicios de urgencias de hospitales del segundo nivel o unidades de medicina familiar, y que fueran enviados a su centro de reperfusión en un hospital de tercer nivel de atención del IMSS. El diseño es un estudio prospectivo consecutivo, no aleatorizado, antes y después, para evaluar los efectos de la implementación del Código Infarto. Los pacientes se dividieron en dos grupos: el grupo I (antes de Código Infarto, 2014) y el grupo II (después de Código Infarto, 2015 y 2016). Se diseñó una red de unidades médicas en la que se incluyeron nueve hospitales y dos unidades de medicina familiar, y enviaban al Hospital de Cardiología del Centro Médico Nacional Siglo XXI, por correo electrónico, chat o llamada telefónica.

Las variables de estudio fueron las estrategias de reperfusión, los tiempos de atención (puerta-aguja y puerta-balón), y las complicaciones durante la hospitalización de falla cardiaca, falla renal, reinfarto, muerte y la combinación de ellas. Los pacientes firmaron el consentimiento bajo información para participar en el estudio.

\section{Análisis estadístico}

La comparación de las variables nominales se hizo con la prueba de ji al cuadrado o con la prueba exacta de Fisher cuando la frecuencia observada era $\geq 5$. Las variables cuantitativas con distribución semejante a la normal se contrastarán con la t de Student para grupos independientes, y las variables cuantitativas con distribución no semejante a la normal con la $U$ de Mann-Whitney. El contraste de hipótesis para diferencia de proporciones se calculó con odds ratio, con intervalos de confianza del $95 \%$ y $p<0.05$. Se utilizó el paquete estadístico SPSS versión 22.

\section{Resultados}

Se estudiaron 1227 pacientes, con una edad promedio de $64 \pm 11$ años, 919 hombres (75\%) y 308 mujeres (25\%). Los pacientes del grupo I fueron 433 (antes de Código Infarto, 2014) y en el grupo II fueron 794 (después de Código Infarto, 2015 y 2016). Fueron enviados de una red de unidades médicas prediseñada, en la que se incluyeron nueve hospitales y dos unidades de medicina familiar, con la coordinación del Hospital de Cardiología del Centro Médico Nacional Siglo XXI, por correo electrónico, chat o llamada telefónica.

Los factores de riesgo se muestran en la tabla 1. Existió diferencia significativa en tabaquismo y dislipidemias; el primero fue más importante en el grupo II, 
mientras que las segundas se presentaron con menor frecuencia en el grupo I. En diabetes e hipertensión no se observó diferencia. Con la implementación de Código Infarto cambiaron significativamente $(p<0.0001)$ las estrategias de reperfusión entre los grupos I y II (Fig. 1). Se redujo en un $26 \%$ el grupo que no recibía TF ni ICP (no reperfundidos), y se mejoró la reperfusión principalmente con un incremento de la ICP del 16.6 al $42.6 \%$, mientras que la TF se redujo del 39.3 al $25 \%$; en este grupo, hasta un $25 \%$ fue llevado a la estrategia farmacoinvasiva.

Los tiempos de atención disminuyeron significativamente: el tiempo puerta-aguja de 92 a 72 minutos $(p=0.004)$ y el tiempo puerta-balón de 140 a $92 \mathrm{mi}-$ nutos $(p<0.0001)$ (Fig. 2).
El estado hemodinámico se muestra en la tabla 2. La fracción de expulsión del ventrículo izquierdo fue menor en el grupo I ( $42 \pm 10 \%$ contra $43 \pm 10 \%$; $p=0.002$ ). Los biomarcadores de necrosis miocárdica de baja especificidad (creatina fosfocinasa y fracción MB) no mostraron diferencia significativa, pero los de alta especificidad fueron más altos en el grupo I (troponina I: 50 contra $23 \mathrm{ng} / \mathrm{dl}, \mathrm{p}=0.009$; mioglobina: 463 contra $299 \mathrm{ng} / \mathrm{dl}, \mathrm{p}=0.005)$. Otros factores de alto riesgo fueron identificados en el grupo I, tales como fibrinógeno (565 contra $524 \mathrm{mg} / \mathrm{dl} ; \mathrm{p}=0.04$ ) y urea (58 contra $53 \mathrm{mg} / \mathrm{dl}$ ). El perfil de lípidos (colesterol total y unido a lipoproteínas de baja y alta densidad, y triglicéridos) y la glucosa no mostraron diferencias entre ambos grupos.

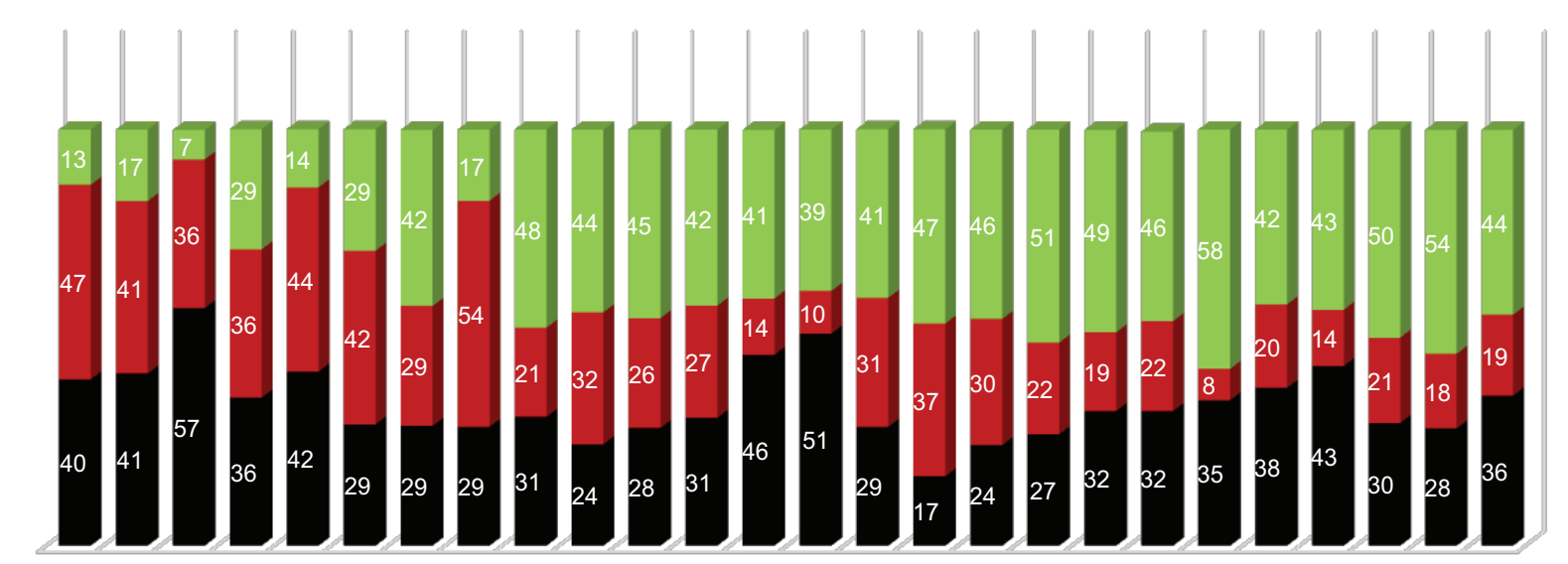

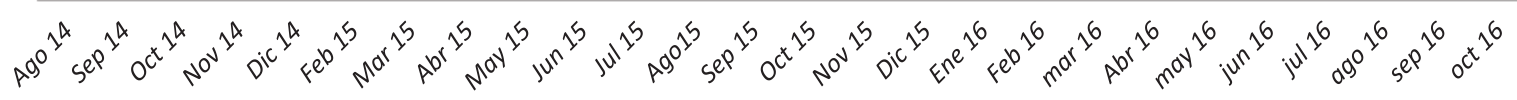

$$
\begin{aligned}
& \text { No reperfundido } \quad \text { terapia fibrinolítica ICP }
\end{aligned}
$$

Figura 1. Cambios en la estrategia de reperfusión antes y después de la implementación del Código Infarto. Se muestra el incremento de la estrategia con intervención coronaria percutánea (ICP) y la reducción de la no reperfusión y de la terapia fibrinolítica.

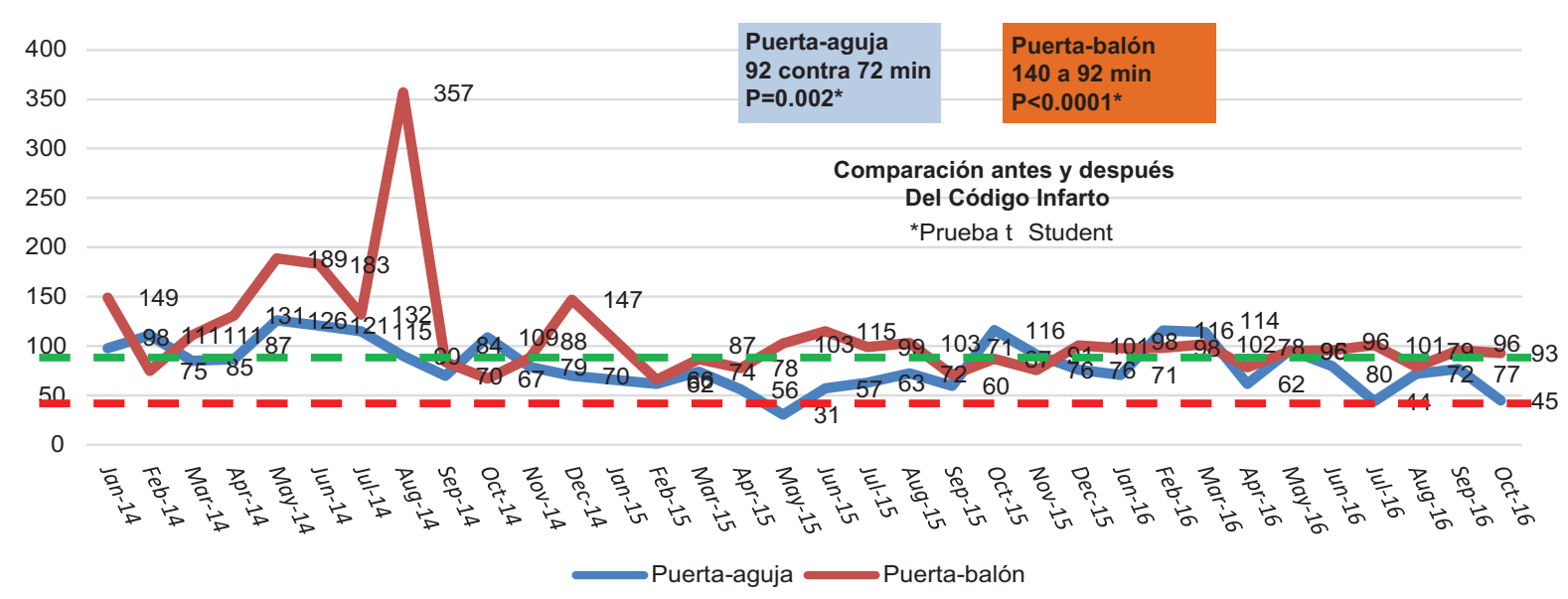

Figura 2. Reducción de los tiempos puerta-aguja y puerta-balón en los pacientes incluidos en la estrategia Código Infarto. 
Tabla 1. Características basales de los pacientes incluidos en la implementación del Código Infarto

\begin{tabular}{|c|c|c|c|}
\hline Descripción & $\begin{array}{c}\text { Grupo I (Antes) } \\
N(\%) \\
433(\%)\end{array}$ & $\begin{array}{c}\text { Grupo II (Después) } \\
\text { N (\%) } \\
794(\%)\end{array}$ & $\mathrm{p}$ \\
\hline Edad & $63.72( \pm 11.02)$ & $63.84( \pm 11.497)$ & 0.896 \\
\hline \multicolumn{4}{|l|}{ Sexo } \\
\hline Masculino & $328(75.8)$ & $591(74.4)$ & NS \\
\hline Femenino & $105(24.2)$ & $203(25.6)$ & NS \\
\hline Total & 433 & 794 & \\
\hline Tabaquismo & $205(47.3)$ & $449(56.5)$ & 0.002 \\
\hline Diabetes mellitus & $197(45.5)$ & $387(48.7)$ & 0.277 \\
\hline Hipertensión arterial sistémica & $264(61.0)$ & $457(57.6)$ & 0.246 \\
\hline Dislipidemia & $202(46.7)$ & $314(39.5)$ & 0.016 \\
\hline Infarto previo & $47(10.9)$ & $109(13.7)$ & 0.149 \\
\hline Angina previa & $45(10.4)$ & $137(11.2)$ & NS \\
\hline
\end{tabular}

Tabla 2. Complicaciones durante la hospitalización en pacientes con infarto agudo de miocardio con elevación del segmento ST

\begin{tabular}{lcccc}
\hline Desenlace & $\begin{array}{c}\text { Grupo I (antes) } \\
\text { N (\%) }\end{array}$ & $\begin{array}{c}\text { Grupo II (después) } \\
\text { N (\%) }\end{array}$ & p & OR (IC 95\%) \\
\hline Insuficiencia cardiaca & $75(17.3)$ & $90(11.3)$ & 0.004 & $0.61(0.43-0.85)$ \\
Reinfarto & $17(3.9)$ & $15(1.9)$ & 0.03 & $0.47(0.23-0.95)$ \\
Muerte & $91(21)$ & $97(12.2)$ & 0.004 & $0.52(0.38-0.71)$ \\
Eventos cardiovasculares mayores & $152(35.3)$ & $233(29.3)$ & 0.034 & $0.76(0.59-0.97)$ \\
\hline
\end{tabular}

IC 95\%: intervalo de confianza del 95\%; OR: odds ratio.

Con la implementación del Código Infarto disminuyeron las complicaciones en el grupo II, tal como se muestra en la tabla 2. Existió menor frecuencia de insuficiencia cardiaca, reinfarto, complicaciones mayores durante la hospitalización (35.3 a 29.3\%; $p=0.034$ ) y muerte $(21$ contra $12 \% ; p=0.004)$. También se observó una reducción significativa de los días de estancia en la unidad de cuidados intensivos (4.3 a 3 días; $p<0.0001$ ), un día menos en hospitalización (6 a 5 días; $p=0.05$ ) y una disminución en la hospitalización total (10 a 8 días; $p=0.02$ ).

\section{Discusión}

En el IAM CEST, la terapia de reperfusión se ha convertido en un predictor de supervivencia a corto y largo plazo ${ }^{11-14}$. El apego a las guías de práctica clínica con la atención regionalizada a través de la implementación del Código Infarto demostró que la terapia de reperfusión reduce de forma importante las complicaciones menores y mayores durante la hospitalización, tanto en insuficiencia cardiaca como en reinfarto y muerte. También se observó que la estandarización del protocolo de atención permitió reducir el porcentaje de pacientes que se quedaban fuera de algún tratamiento de reperfusión, incrementándose la tasa ICP, lo cual impacta en la reducción de las complicaciones. Estas cifras son comparables a las de los estudios iniciales de la TF y el ICP (GISSII5, ISIS- ${ }^{16}$, Maastricht ${ }^{17}$ ), en los que se observó una importante reducción en la mortalidad y las complicaciones.

La estrategia farmacoinvasiva, es decir, después de la TF exitosa realizar la ICP transcurridas entre 3 y 24 horas, puede ser una opción para nuestro país, ya que en el proceso de implementación se optó en algunos casos por la TF al no contar con la disponibilidad de la sala de hemodinámica en los primeros 90 minutos del contacto (posteriormente los pacientes se enviaron a la sala $)^{18}$.

La estrategia Código Infarto es la primera en su género en nuestro país. Se pretende implementar al menos en 12 redes más con el mismo protocolo de 
atención, con la finalidad de estandarizarlo. Se hará un seguimiento puntual de cada caso y se verá cuáles son las áreas de oportunidad para mejorar el proceso.

\section{Conclusiones}

La implementación del Código Infarto permitió mejorar las estrategias de reperfusión en el IMSS, reduciendo los tiempos puerta-aguja y puerta-balón de manera significativa. También se observaron menos complicaciones tempranas y menos muerte. Sin embargo aun se deben reducir los tiempos de atención y el porcentaje de nos reperfundidos.

\section{Agradecimientos}

\section{A la Fundación IMSS.}

\section{Bibliografía}

1. Organización para la Cooperación y el Desarrollo Económicos (OCDE) 2015. Disponible en: http://dx.doi.org/10.1787/health-data.en

2. Instituto Nacional de Estadística, Geografía e Informática. Epidemiología de la defunción poblacional. México: INEGI; 2015. Disponible en: www. inegi.gob.mx

3. Keeley EC, Boura JA, Grines CL. Primary angioplasty versus intravenous thrombolytic therapy for acute myocardial infarction: a quantitative review of 23 randomized trials. Lancet. 2003;361:13-20.

4. Steg PG, Bonnefoy E, Chabaud S, et al. Comparison of Angioplasty and Prehospital Thrombolysis In acute Myocardial infarction (CAPTIM) Investigators. Impact of time to treatment on mortality after prehospital fibrinolysis or primary angioplasty: data from the CAPTIM randomized clinical trial. Circulation. 2003:108:2851-6.

5. Pinto DS, Kirtane AJ, Nallamothu BK, et al. Hospital delays in reperfusion for ST elevation myocardial infarction: implications when selecting a reperfusion strategy. Circulation. 2006;114:2019-25.
6. The task force on the management of ST-segment elevation acute myocardial infarction of European Society of Cardiology (ESC). ESC Guidelines for the management of acute myocardial infarction in patients presenting with ST-segment elevation. Eur Heart J. 2012; 33:2569-619.

7. 2014 AHA/ACC Guideline for the management of patients with non-ST-elevation acute coronary syndromes. A report of the American College of Cardiology/American Heart Association Task Force on Practice Guidelines. Circulation. 2014;130:e344-e426.

8. Borrayo-Sánchez G, Madrid-Miller A, Arriaga-Nava R, et al. Riesgo estratificado de los síndromes coronarios agudos. Resultados del primer RENASCA-IMSS. Rev Med Inst Mex Seguro Soc. 2010;48:259-64.

9. Xavier D, Pais P, Devereaux PJ, et al., for the TN-STEMI Programme Investigators. CREATE registry investigators. Treatment and outcomes of acute coronary síndromes in India (CREATE): a prospective analysis of registry data. Lancet. 2008;371:1435-42.

10. Borrayo SG, Martínez MOG, Gudiño TA, et al. Código Infarto. Protocolo para servicios de urgencias. México 2015. Primera edición. ISBN Obra independiente: 978-607-8392-42-1.

11. Krumholz HM, Wang $Y$, Mattera JA, et al. An administrative claims model suitable for profiling hospital performance based on 30-day mortality rates among patients with an acute myocardial infarction. Circulation. 2006; 113:1683-92.

12. Seghieri C, Mimmi S, Lenzi J, et al. 30-day in-hospital mortality after acute myocardial infarction in Tuscany (Italy): an observational study using hospital discharge data. BMC Med Res Methodol. 2012;12:170.

13. Ross JS, Maynard C, Krumholz HM, et al. Use of administrative claims models to assess 30-day mortality among Veterans Health Administration hospitals. Med Care. 2010;48:652-8.

14. Hayashida K, Imanaka Y, Sekimoto M, et al. Evaluation of acute myocardial infarction in-hospital mortality using a risk-adjustment model based on Japanese administrative data. J Int Med Res. 2007;35:590-6.

15. Gruppo Italiano per lo Studio Della Streptochinasi nell'Infarcto Miocardio (GISSI). Effectiveness of intravenous thrombolytic treatment in acute myocardial infarction. Lancet. 1986;1:397-402.

16. ISIS-2 (Second International Study Of Infarct Survival) Collaborative Group. Randomized trial of intravenous steptokinase, oral aspirin, both or neither among 17,187 cases of suspected acute myocardial infarction; ISIS-2. Lancet. 1988;2:349-60.

17. Vermeer F, Oude Ophuis AJM, van den Berg, et al. Prospective randomized comparison between thrombolysis, rescue PCTA, and primary PTCA in patients with extensive myocardial infarction admitted to a hospital without PTCA facilities: a safety and feasibility study. Heart. 1999;82:426-31.

18. Larson DM, Duval S, Sharkey SW, et al. Safety and efficacy of a pharmaco-invasive reperfusion strategy in rural ST-elevation myocardial infarction patients with expected delays due to long-distance transfers. Eur Heart J. 2012;33:1232-40. 